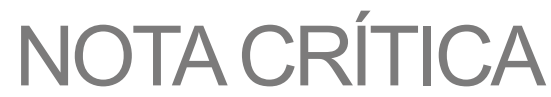

\section{REGULADORES \\ Y SUPERVISORES \\ ECONÓMICOS \\ INDEPENDIENTES: \\ EL CASO ESPAÑOL}

\section{Julio Segura, Coord.}

Editorial Centro de Estudios Ramón

Areces, 2018

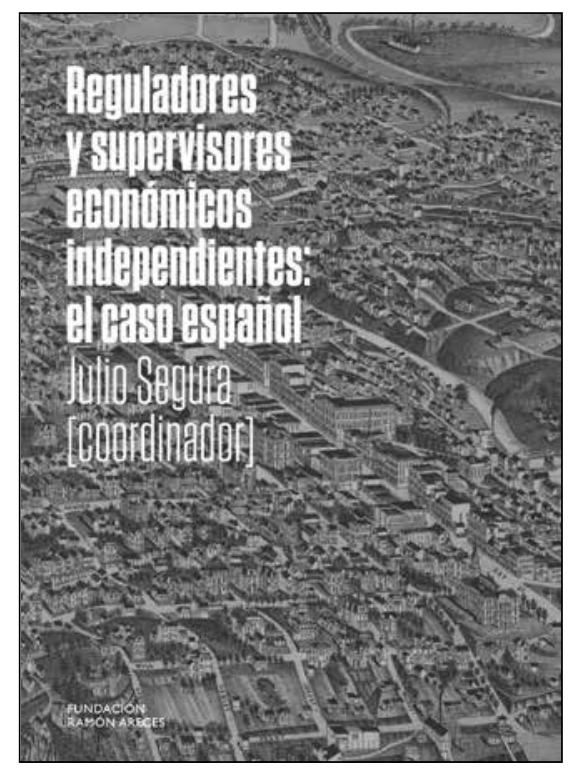

La calidad institucional es clave para el crecimiento económico de un país a largo plazo, como ponen de manifiesto diversos desarrollos teóricos y notable evidencia empírica. Los organismos independientes (donde los Gobiernos carecen de control y dirección) son una de esas instituciones esenciales, pues permiten desligar del ciclo político la regulación y supervisión de sectores (como el financiero, la energía o las telecomunicaciones) y ámbitos (como la defensa de la competencia, la responsabilidad fiscal o las cuentas públicas) clave para el buen desempeño de la economía (de forma que se asegure la consistencia temporal de la intervención del sector público). Por ello la independencia y la eficacia con las que estos organismos desarrollan su tarea es vital para un óptimo funcionamiento de los sectores y ámbitos supervisados y por ende para el conjunto de la economía. Pero son igualmente relevantes la transparencia y la rendición de cuentas, para disipar las dudas sobre la legitimidad que puedan surgir por su independencia de Gobiernos elegidos democráticamente.

El libro reseñado aborda la aplicación de todos estos aspectos (independencia, eficacia, transparencia y rendición de cuentas) a los reguladores y supervisores independientes en el caso español. El primer capítulo (a cargo de Julio Segura) analiza, a modo de introducción, el papel de los organismos independientes en las economías avanzadas. Los siguientes capítulos (del 2 al 7) se dedican a los ámbitos (anteriormente mencionados) de defensa de la competencia (a cargo de Pilar Sánchez), supervisión financiera (Julio Segura), responsabilidad fiscal (Miguel Ángel García Díaz y Federico Geli), energía (Miguel Ángel Lasheras Merino), telecomunicaciones (Juan Delgado) y control de cuentas públicas (a cargo de Elisa de la Nuez SánchezCasado y Carlota Tarín). Los seis capítulos describen la evolución de las respectivas instituciones en España y cuentan con conclusiones y recomendaciones en aras de mejorar el papel de estos organismos. El octavo y último capítulo (a cargo de Julio Segura) recopila precisamente las conclusiones generales que se pueden extraer de manera transversal. Esto convierte al libro en lectura obligada para policy-makers, tanto para los responsables de poderes públicos (ejecutivo y legislativo) como para los propios gestores de organismos reguladores y supervisores.

Esta nota se centra principalmente en los capítulos de defensa de la competencia, energía y telecomunicaciones, por su mayor interés para los lectores de este monográfico. Pero recomienda encarecidamente la lectura de todo el libro.

El capítulo de defensa de la competencia (a cargo de Pilar Sánchez), tras realizar una reseña histórica de la evolución de esta política en las principales jurisdicciones, se adentra en debates profundos. Uno de ellos atañe a los fines de la política de competencia. La salvaguarda de la competencia y de la eficiencia en el mercado (cuyo estándar general es el bienestar del consumidor) puede implicar otros fines auxiliares como la protección 
del consumidor, la defensa de los competidores (especialmente pymes) y del proceso competitivo (incluyendo un «campo de juego equilibrado», level playing field) e incluso la lucha contra la desigualdad por sus vínculos con la contratación empresarial (aludiendo, aunque sin mencionarlo, a las propuestas más heterodoxas que emanan del denominado movimiento hipster antitrust). La autora de este capítulo propone una mejor definición de los fines de la normativa de competencia en la legislación española.

Este capítulo aborda también aspectos más instrumentales. En este caso, la autora sí concluye que España cuenta con un aparato suficiente y homologable a las principales jurisdicciones. En primer lugar, el control de conductas, incluyendo relevantes herramientas formales (como el programa de clemencia, las inspecciones o la terminación convencional) y de fondo (como el análisis económico, cuando las conductas no son sancionables per se). Aunque para una mayor efectividad de este control es fundamental el carácter disuasorio de las sanciones y en España es clave una modificación normativa que asegure que el límite de la multa del 10 por 100 de la cifra de negocios global de la empresa no se module en función de la gravedad de la infracción (pues en términos comunitarios la imposición de ese umbral solo obedece a la salvaguarda financiera de la empresa). Otro aspecto que puede contribuir a la disuasión en España es la reclamación de daños, pues otras medidas más agresivas, como la criminalización de los cárteles con penas de cárcel, no son viables por la utilización de la vía administrativa.

En cualquier caso, la autora advierte de manera pertinente de los retos que supone la economía digital para la detección y sanción de conductas anticompetitivas. También para el segundo instrumento como es el control de concentraciones, donde puede caber una adaptación de ciertos aspectos a la digitalización, por ejemplo, mediante una rebaja o modificación de umbrales y criterios de notificación.

El tercer instrumento es el control de las ayudas públicas, un ámbito esencialmente comunitario donde la autoridad española tiene competencias de promoción. En cuarto lugar, está la política de promoción de la competencia, donde se puede considerar que la autoridad española de hecho cuenta con una actividad por encima de sus homólogos internacionales, con notables informes sobre normativa, estudios y guías, y con un potente y novedoso instrumento como es la legitimación activa (la posibilidad de recurrir ante la jurisdicción competente actos y normas con rango inferior a ley que impliquen restricciones a la competencia). Sin embargo, la autora echa en falta un mayor incentivo al uso de los programas de cumplimiento normativo que reduzcan los costes del uso de herramientas punitivas y creen una mayor cultura de competencia.

Más controvertido que el aparato instrumental es el diseño institucional. Actualmente existe una tendencia a la integración de organismos por ahorros de costes, coordinación, sinergias y eficiencias. Este proceso ha afectado sobre todo a la defensa de la competencia y la protección del consumidor, como en el caso de Reino Unido. Otros países, como España con la Comisión Nacional de los Mercados y la Competencia (CNMC), han optado por integrar defensa de la competencia y regulación sectorial. $\mathrm{Y}$ otros, como Países Bajos, las tres áreas: defensa de la competencia, protección del consumidor y regulación sectorial. La autora identifica oportunidades de esta tendencia: complementariedad de objetivos (especialmente en competencia y protección del consumidor), ganancias de eficiencia, menor riesgo de captura (por la multitud de objetivos), seguridad jurídica (por la toma de decisiones únicas), etc. Pero también riesgos: integración de organizaciones y distintas culturas de recursos humanos, dificultad de priorizar objetivos, etc. La autora es crítica con la reforma de 2013 de integración institucional en la CNMC, pero también con 
las posibles reformas que se han anunciado de revertir esa integración. En ambos casos, por no sopesar de manera suficiente la validez del modelo vigente.

En cambio, la autora sí refrenda el modelo administrativo de agencia integrado con fases de instrucción/investigación y resolución en el mismo organismo, aunque con separación total (modelo no afectado por la reforma de 2013). Este modelo integrado brinda eficiencias procedimentales y de especialización sin perjuicio de los derechos de las partes, pues las decisiones se pueden someter a revisión por jurisdicción ordinaria (incluso por el Tribunal Supremo). De hecho, la autora plantea la posibilidad de que la revisión jurisdiccional se haga por salas especializadas o, al menos, con equipos de trabajo especializados.

Sin perjuicio de la relevancia del resto de aspectos ya mencionados, la autora estima que el atributo más relevante es la independencia de la institución. $Y$ para ello son necesarias dos cuestiones. La primera es la independencia orgánica, funcional y presupuestaria, pudiéndose alcanzar esta última con una financiación en función de tasas sobre concentraciones y un porcentaje de las multas. La segunda es la independencia de los miembros de sus órganos decisorios que (aparte de con la imposibilidad de cese salvo causa grave justificada) se consigue con un procedimiento de selección que asegure el conocimiento, la experiencia y el prestigio (especialización que puede permear también al resto de recursos humanos si se opta por un procedimiento de acceso más flexible que el existente para funcionarios de carrera).

El capítulo de energía (a cargo de Miguel Ángel Lasheras Merino) parte de la historia reciente de los reguladores independientes en este ámbito sectorial, tanto a nivel supranacional como nacional. El autor estima que los avances en independencia del regulador energético han sido muy tímidos y siempre por impulso comunitario más que por convencimiento nacional. Pero esta independencia es crucial para la consistencia temporal de la regulación sectorial (en este caso, de la energía), de forma que se asegure una retribución suficiente a los operadores para incentivar sus inversiones sin tener la tentación de reducir dicha retribución por motivos electoralistas o de ciclo político.

Por ello la competencia fundamental de un regulador independiente habría de ser la determinación de tarifas, peajes y precios regulados. Pero la energía es un sector particular por diversos motivos (como de manera incisiva señala el autor citando a diversos ministros), como su peso en el gasto de empresas y hogares o la volatilidad de precios debida a factores exógenos (como el clima o la coyuntura internacional en mercados de materias primas). Eso ha hecho que la regulación se haya mantenido en el Gobierno, generando diversos problemas.

El primero, el de los costes de transición a la competencia (CTC), con una remuneración incierta y no ajustada al coste y al peso respectivo de los operadores entre 1997 y 2010.

El segundo, y más relevante, el déficit de tarifa, con unos precios que no recogían todos los incrementos de costes reconocidos por la regulación (problema que ha existido desde el año 2000). Inicialmente ese incremento de costes estuvo causado parcialmente también por los CTC. Pero la explosión de costes, y por tanto del déficit de tarifa, llegó a partir de 2005 , señalando el autor especialmente la retribución a las energías renovables (por unas primas generosas y/o por la no limitación de la cantidad de energía primada). Este problema no se ha atajado en su núcleo hasta 2013 con un recorte de ingresos a los generadores.

El tercero es el «bono social» en sus distintas formas (tarifa de último recurso, TUR, o más adelante precio voluntario al pequeño consumidor, PVPC); esto es, una 
tarifa subvencionada para proteger a consumidores vulnerables de alzas excesivas. Las desavenencias entre regulador y Gobierno se han debido a cuestiones de fondo (pues el regulador pide proteger a los consumidores en función de su renta y el Gobierno ha optado por potencia contratada) y de forma (pues el regulador defiende disponer de las competencias para determinar la metodología y aprobar las tarifas).

El cuarto problema detectado por el autor es la ausencia de peso del regulador en cuestiones de competencia, especialmente en las fusiones y adquisiciones que se anunciaron o se produjeron fundamentalmente en la década de los años 2000.

Por todo ello, el autor concluye (de manera similar al capítulo de competencia) que la independencia y profesionalidad (tanto de los dirigentes como del personal técnico) del regulador deben jugar un papel fundamental en el futuro.

El capítulo de telecomunicaciones (a cargo de Juan Delgado) parte de una contextualización teórica e internacional de la liberalización en España. En España se dieron ciertos condicionantes específicos en los inicios del proceso, como un uso de la privatización y la liberalización para cumplir objetivos presupuestarios y de inflación. El regulador de telecomunicaciones ha ido acompañando ese proceso de liberalización en los distintos segmentos del mercado: telefonía fija (con una liberalización total), banda ancha (liberalizado aunque con obligación de pasar un test de replicabilidad de las ofertas minoristas para el operador con poder significativo de mercado) y telefonía móvil (liberalizada antes que la fija por la mayor competencia asociada a los menores requisitos tecnológicos para la entrada).

Pero ha habido muchos retos en el camino, y el autor destaca los conflictos entre los objetivos de competencia y los de regulación. Por ejemplo, la sanción de la Dirección General de Competencia de la Comisión Europea a Telefónica (ratificada por el Tribunal de Justicia de la Unión Europea) por sus precios de banda ancha previamente aprobados por la Comisión del Mercado de las Telecomunicaciones (CMT). También sanciones de la CMT a operadores por conductas contrarias a la normativa de competencia, que el Tribunal Supremo desautorizó por considerar fuera de su ámbito de actuación. $Y$ otras discrepancias entre la Autoridad de Competencia (Comisión Nacional de Competencia, CNC) y la CMT por la aplicación de la regulación sectorial.

Sin embargo, el autor considera que el modelo de integración institucional de la CNMC no soluciona esos conflictos, que siguen existiendo entre la Sala de Supervisión Regulatoria y la Sala de Competencia, pero que ahora se dirimen de manera más opaca dentro de la misma institución.

El autor también critica el crecimiento (que podríamos denominar «externo») del regulador de comunicaciones con la integración de otros sectores. Esa multisectorialidad puede tener sentido con el sector postal o el audiovisual, por sus relativas sinergias sectoriales. Pero el autor arguye que estos puntos comunes son muy limitados con sectores como el transporte y la energía, y critica la imposibilidad de especialización de la Sala de Supervisión Regulatoria de la CNMC ante asuntos tan variados.

Además, el autor apunta que en algunos ámbitos de transporte (como en el ferroviario) y energía (como en electricidad) el grado y éxito de la liberalización es mucho menor que en el sector de las telecomunicaciones. Por su parte, los retos regulatorios actuales de las telecomunicaciones (como la convergencia tecnológica, la gestión del espectro, la neutralidad de red, la interoperabilidad y el auge de proveedores de contenidos independientes u over-the-top, OTT) responden a una evolución de la tecnología más dinámica. Esos retos de las telecomunicaciones hacen 
abogar al autor por otro tipo de crecimiento (que podríamos denominar «interno») del regulador de telecomunicaciones, asumiendo más competencias dentro de su sector y de sectores conexos: la gestión de Internet y regulación del comercio electrónico, la gestión del espectro, la protección del consumidor, la defensa de la competencia, etc.

El autor también apunta a los retos que ha supuesto la inclusión de la CMT en la CNMC en términos de pérdida de independencia presupuestaria y el manejo de su organización interna y de su política de personal (salarios y dimensión de la plantilla). En relación a los recursos humanos, la convivencia de dos regímenes de personal distintos también genera fricciones e impide aprovechar sinergias por la movilidad interna. $Y$ el autor estima que el régimen de funcionario resta independencia, pues se tomarían menos resoluciones contrarias al Ejecutivo para no perjudicar la carrera profesional en un eventual retorno a un centro directivo de la Administración.

El autor también recuerda otros problemas de la integración institucional, como el pronunciamiento del Tribunal Supremo (incluyendo una cuestión prejudicial resuelta por el Tribunal de Justicia de la Unión Europea) acerca de los ceses de miembros del Consejo de la CMT, fruto de la creación de la CNMC, considerándolos un riesgo para la independencia del regulador.

No obstante, el autor también recuerda otras innovaciones positivas aportadas por la CNMC, como es el refuerzo de la legalidad, la rendición de cuentas y la transparencia a través de la creación del Departamento de Control Interno o de un registro de grupos de interés.

El autor considera que el diseño institucional no es decisivo, aunque aboga por la especialización de los órganos de resolución en mayor o menor medida. $Y$ también recuerda (con más énfasis que en otros capítulos) que la independencia no es un fin en sí mismo sino un medio para hacer efectiva la regulación. Ha de ir acompañada de la capacidad técnica de sus directivos y el personal (aspectos también enfatizados por los otros capítulos), de una organización interna adecuada y de una aplicación óptima de un marco regulatorio apropiado.

En definitiva, enlazando estos capítulos específicos con las conclusiones y recomendaciones generales (a cargo de Julio Segura), los autores destacan, entre otros, dos problemas comunes para el caso español: inadecuado sistema de nombramiento y selección de dirigentes y falta de independencia económico-financiera y organizativa. Se trata de opiniones aventuradas pero que son valientes y provienen de fuentes autorizadas. Por tanto, deben ser como mínimo tenidas en cuenta por los responsables del diseño de estos organismos independientes. En la calidad de sus instituciones España se juega su futuro.

Pedro Hinojo 